\title{
Patients' Serum and Urine as Easily Accessible Samples for the Measurement of Non-Vitamin K Antagonist Oral Anticoagulants
}

\author{
Job Harenberg, MD ${ }^{1}$ Shanshan Du, $\mathrm{PhD}^{1}$ Sandra Krämer, $\mathrm{MD}^{1}$ Christel Weiss, $\mathrm{PhD}^{2}$ \\ Roland Krämer, $\mathrm{PhD}^{3}$ Martin Wehling, $\mathrm{MD}^{1}$
}

${ }^{1}$ Clinical Pharmacology, Medical Faculty Mannheim, Ruprecht-Karls University Heidelberg, Mannheim, Germany

2 Biometry and Statistics, Medical Faculty Mannheim, Ruprecht-Karls University Heidelberg, Mannheim, Germany

${ }^{3}$ Institute Inorganic Chemistry, University of Heidelberg,

Heidelberg, Germany

Semin Thromb Hemost 2015;41:228-236.

\begin{abstract}
Address for correspondence Job Harenberg, MD, Clinical Pharmacology, Medical Faculty Mannheim, Ruprecht-Karls University Heidelberg, Maybachstrasse 14, 68169 Mannheim, Germany (e-mail: job.harenberg@medma.uni-heidelberg.de).
\end{abstract}

\begin{abstract}
Measurement of the anticoagulant effect of non-vitamin $\mathrm{K}$ antagonist oral anticoagulants (NOAC) may be desirable, in particular in patients with acute medical conditions. Useful methods should give results rapidly within minutes, should be easy to perform, specific, and sensitive. Using plasma samples, chromogenic assays can be made to be specific for the two types of NOAC (factor Xa and thrombin inhibitors), and also hemoclot and ecarin clotting time specific for dabigatran. If plasma

Keywords

- direct factor Xa inhibitors

- direct thrombin inhibitors

- new oral direct anticoagulant

- serum

- urine

- plasma

- chromogenic substrate methods samples anticoagulated with sodium citrate are not available, blood samples anticoagulated with ethylene diamine tetraacetic acid or serum samples may be regarded as alternatives for the determination of NOAC. At present, dabigatran cannot be determined from serum samples because it may be consumed during the clotting process to obtain serum. NOAC can be determined in urine samples due to their renal elimination. Quantitative methods are preferable to qualitative methods, although the latter may be advantageous in some situations, being developed as point-of-care tests for oral factor $\mathrm{Xa}$ and thrombin inhibitors. In these tests, the presence and absence of NOAC in urine can be identified with the naked eye after a few minutes and these tests are highly specific and sensitive. New assays such as a semiquantitative determination in urine samples and measurement using other sample matrices are currently under development.
\end{abstract}

Besides the conventional vitamin $\mathrm{K}$ antagonists, the nonvitamin $\mathrm{K}$ antagonist oral anticoagulants (NOAC) such as dabigatran, rivaroxaban, apixaban, and edoxaban are approved in many countries for the prevention of venous and arterial thromboembolism. ${ }^{1}$ All compounds were developed with the dual aims of avoiding laboratory monitoring and to be used at fixed daily dose. As a consequence, plasma samples for post hoc determination of the anticoagulant effect were not taken in the pivotal studies or only assessed in subgroups of patients.
One of the common characteristics of these anticoagulants is the considerable, though differing, impact of renal function on their elimination. Dabigatran is excreted by the kidneys to approximately $80 \%$, rivaroxaban to $\sim 60 \%$, of which one half represents the active drug, and apixaban and edoxaban to $\sim 25 \%$. Dabigatran is therefore contraindicated in patients with a creatinine clearance below $30 \mathrm{~mL} / \mathrm{min}$, and the factor Xa inhibitors at below $15 \mathrm{~mL} / \mathrm{min}^{2}$. For intermediate decreases of renal function,
Issue Theme Anticoagulant Therapy: Present and Future; Guest Editor: Job Harenberg, MD. published online

February 15, 2015
DOI http://dx.doi.org/ 10.1055/s-0035-1544158. ISSN 0094-6176.
Copyright (c) 2015 by Thieme Medical Publishers, Inc., 333 Seventh Avenue, New York, NY 10001, USA. Tel: +1(212) 584-4662.
License terms

(๑) $\odot \circledast$ 
individual reduction of doses of rivaroxaban, apixaban, and edoxaban are required. In the clinical studies, patients with impaired renal function with a creatinine clearance below these limits were not included.

In real-world situations after market approval, NOACs are administered to patient types not included into the clinical studies, and patients may develop diseases over time or take additional drugs (e.g., nonsteroidal anti-inflammatory drugs) that result in reduction of renal function. Patients may also have to undergo acute surgical interventions or may receive drugs interacting with NOAC metabolism at the level of the cytochrome P450 3A4 system or with its transmembrane transport (e.g., in the gut) at the level of P-glycoprotein. These and other clinical situations lead to the inevitable fact that laboratory testing of NOAC will be required in specific clinical situations in the future. Importantly, these clinical situations most often require urgent medical decisions necessitating rapid monitoring. Such rapid monitoring is feasible specifically with point-of-care (POC) methods. Conventional methods, POC methods, and alternative matrices for determination of NOACs are reviewed in this article (see - Fig. $\mathbf{1}$ ).

\section{Liquid Chromatography Methods}

Liquid chromatography with high-resolution mass spectrometry (LC-MS/MS) methods were used initially for the development of the NOAC. They were adopted to identify the purity of the compounds during the production process as well as for the identification of metabolites following administration to animals and humans. However, the availability of these methods is limited to a small number of specialized laboratories. ${ }^{3,4}$ Recently, these methods were used to compare the sensitivity and specificity of chromogenic substrate assays and of coagulation assays for NOACs after spiking to human plasma or after administration to man. ${ }^{5}$ The most relevant conclusion of those investigations is that the LC-MS/MS methods show a strong correlation to the specific chromogenic substrate assays after spiking human plasma samples with dabigatran, rivaroxaban, apixaban, and edoxaban, as well as to a specific clotting test for dabigatran (Pearson correlation $r>0.95$ ). Following administration of these NOAC to volunteers or patients, correlations were also very strong with values of $r>0.90$. Consequently, not all studies used LC-MS/MS method as reference to measure NOAC from human plasma samples but used the specific chromogenic substrate assays for the oral direct factor Xa and thrombin inhibitors.

LC-MS/MS results also demonstrated the limited utility of global coagulation assays such as prothrombin time (PT) and activated partial thromboplastin time (aPTT) assays including different reagents for both type of assays. For other coagulation assays, such comparisons were not performed. Because of the strong correlation of the LC-MS/MS methods with chromogenic substrate assays, comparisons of the anticoagulant effects of NOAC with other clotting assays were reported in comparison with the specific chromogenic substrate assays. ${ }^{3,4,6-8}$

A limitation of the LC-MS/MS methods ${ }^{3}$ is that they differ in the experimental setting (e.g., turbulent chromatography ${ }^{6}$ and ultra-performance liquid chromatography-tandem mass spectrometry [UPLC-MS/MS] $)^{7}$ and that these specific methods were not compared.

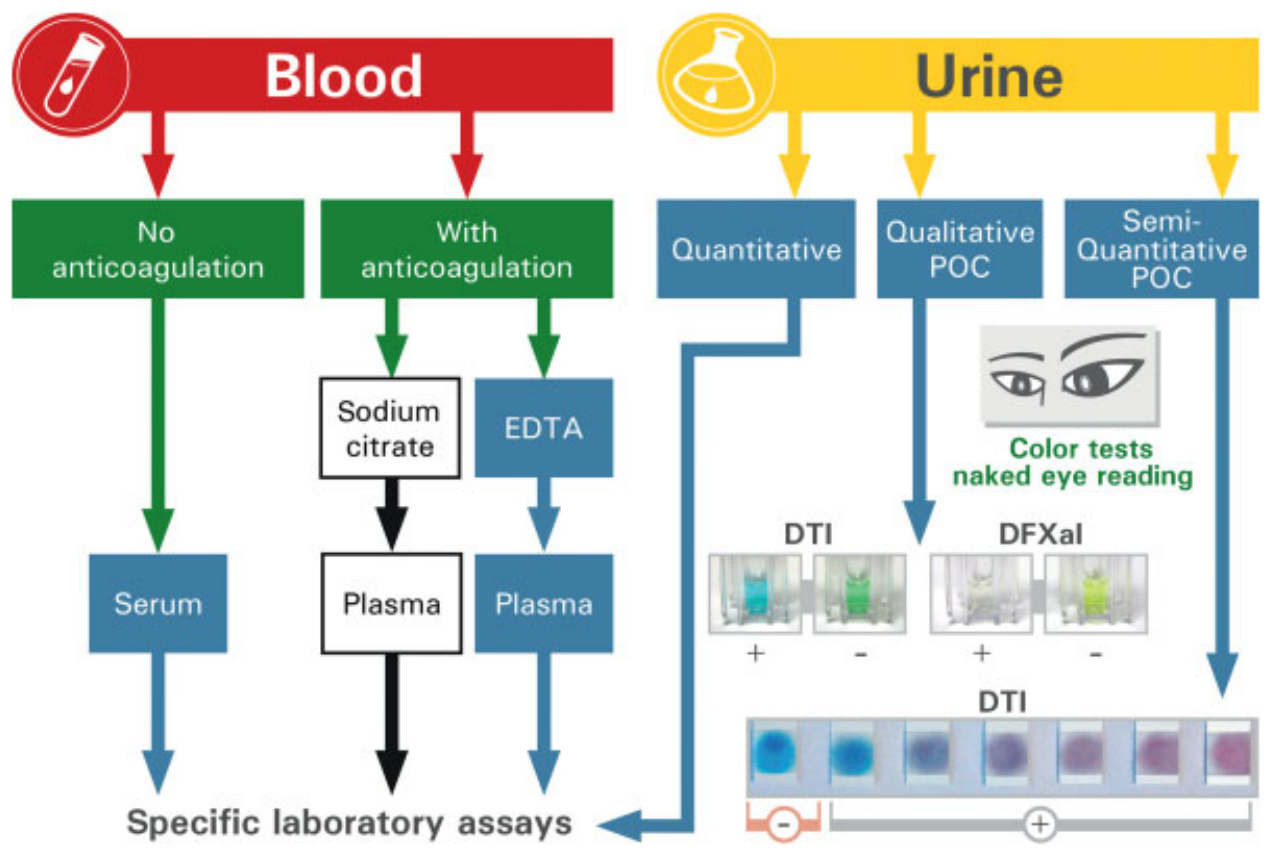

Fig. 1 Outline of the matrices and methods to measure NOAC. Identification of colors: red and yellow, color of the matrix (blood and urine); green, anticoagulation ex vivo during blood sampling; no color, conventional matrix (citrated blood samples); blue, new matrices for measurement of NOAC. POC tests for DTI (direct thrombin inhibitor: dabigatran and others) and DFXal (direct factor Xa inhibitors: rivaroxaban, apixaban, edoxaban and others): a negative (right side) and a positive (left side) tests for the absence or presence of DTI and DFXal in urine samples are shown. The colors can be identified by the naked eye within 10 to 15 minutes. Test details are described in Du et al. ${ }^{18}$ 


\section{Influence of Coagulation Platforms on Coagulation Assays}

Chromogenic substrate assays and coagulation assays are performed on a variety of coagulation platforms. Every assay needs to be adapted to a specific coagulation platform. An example is given here: human plasma samples spiked with different concentrations of apixaban were investigated in an international collaborative study on factor Xa-specific chromogenic substrate methods, such as STA Liquid Anti-Xa (Diagnostica Stago, Asnières sur Seine, France), STA Rotachrom (Diagnostica Stago), Coamatic Heparin (Haemochrom, Essen, Germany), HemosIL Anti-Xa liquid (Instrumentation Laboratory, Kirchheim, Germany), and Technochrome Anti-Xa (Technoclone, Vienna, Austria). ${ }^{9}$ Each laboratory used its own coagulation platform. Subanalysis and comparison of data were done for centers using the ACL coagulation platform (Instrumentation Laboratory) versus centers not using the ACL coagulation platform, and also for centers using the Stago coagulation platform versus centers not using the Stago coagulation. As a result, the coefficient of variation (CV) of the data was lower for all concentrations of apixaban if reagents and platforms were from the same producer compared with the data of centers using the chromogenic assays on platforms of another producer. In detail, the chromogenic assays HemosIL and Coamatic (Instrumentation laboratory) resulted in a lower $\mathrm{CV}$ on the ACL platform compared with the other platforms. The STA-Liquid Anti-Xa and Rotachrom chromogenic assays (Diagnostica Stago) resulted in a lower CV when analyzed on the Stago coagulation platform compared with the other coagulation platform. ${ }^{10}$ Similar data can be anticipated for coagulation assays when they are performed on coagulation platforms on which the coagulation assays were developed. These results show the importance of analyzing and describing each assay for each coagulation platform. In addition, the data show that assays perform better if they are used on the coagulation platform on which the analysis has been optimized by specific adaptations of the experimental setting. These results also show that the variability of measurements of NOAC concentrations or activities may result from such differences in analytical techniques used in the publications.

\section{Chromogenic Assays for Oral Direct Thrombin Inhibitors}

The direct thrombin inhibitor (DTI) dabigatran is a small synthetic oral anticoagulant with a nonpeptidic, potent, specific, competitive, and reversible binding to free and fibrin-bound thrombin. ${ }^{11}$ Dabigatran etexilate is rapidly hydrolyzed to its active form dabigatran after oral administration and intestinal absorption by nonspecific, ubiquitous esterases in gut, blood, and liver. ${ }^{12}$ To improve oral absorption, tartaric acid is added to the oral preparation.

\section{Direct Thrombin Inhibitors Assay}

One of the thrombin specific chromogenic substrate methods is the DTI assay (Siemens Healthcare Diagnostics Inc., Marburg,
Germany). This assay was initially developed and certified for hirudins. New studies showed the applicability of the assay for samples containing dabigatran following spiking to human plasma and after administration to patients treated with dabigatran. ${ }^{13}$ The concentrations of samples were calculated from control samples containing known concentrations of dabigatran. $5,9,14$

\section{S2238 Chromogenic Method}

The chromogenic substrate S2238 is another substrate specific for the determination of any thrombin inhibitor. It has also been used for the measurement of dabigatran in plasma samples spiked with dabigatran and form patients on treatment with dabigatran. As outlined above, some differences in the concentrations in plasma samples may be detected due to the development of each chromogenic substrate assay for a specific coagulation platform. Preliminary data seem to support this finding, which has been reported in more detail for apixaban and various chromogenic methods on different coagulation platforms. ${ }^{13}$

\section{Ecarin Chromogenic Assay}

Ecarin is a snake venom isolated from Echis carinatus; it is used to convert prothrombin to meizothrombin, a prothrombin intermediate that is sensitive to inhibition by DTIs. ${ }^{15,16}$ Ecarin actives factor $\mathrm{V}$ in the plasma sample resulting in a conversion of prothrombin to thrombin. Thereby, thrombin is not added to this type of chromogenic assay. A thrombinspecific chromogenic substrate is released dose dependently by thrombin and inhibited by dabigatran inversely to the concentration of the inhibitor. This assay is specific for dabigatran but is not presently used as frequently as other chromogenic substrate assays for the determination of dabigatran.

\section{Clotting Time Assays for Oral Direct Thrombin Inhibitors}

\section{Hemoclot Thrombin Inhibitor Assay}

The procedure of hemoclot thrombin inhibitor assay (HTI) (HYPHEN BioMed, Neuville-sur-Oise, France) is a diluted thrombin clotting time method. Plasma is diluted 1 to 8 with human pool plasma and thrombin is added to induce clotting. The clotting times are prolonged by low ( $\sim 10$ to 20 $\mathrm{ng} / \mathrm{mL})$ to high $(\sim 500 \mathrm{ng} / \mathrm{mL})$ concentrations of dabigatran in a linear fashion. ${ }^{17}$ This assay was introduced as a standard method by the US Food and Drug Administration (FDA) to determine dabigatran. In fact, many publications reported on the high correlation to the LC-MS/MS method and to chromogenic methods and on the usefulness in patients. However, in patient samples, a high variation of the results was observed, a finding that was attributed mainly to the variation of the pharmacokinetics of dabigatran, and patients' adherence to therapy rather than to the performance of the assay. Using specific calibrators, the clotting times are converted to $\mathrm{ng} / \mathrm{mL}$ dabigatran per $\mathrm{mL}$ plasma. 


\section{Ecarin Clotting Time}

Ecarin is snake venom isolated from Echis carinatus as described above. The ecarin reagent (Pentapharm, Basle, Switzerland) is added to initiate clotting. The clotting times are linearly prolonged by dabigatran, and through calibrators the concentrations of dabigatran are calculated. As for the ecarin chromogenic assay, this test is presently also not used frequently for experimental analyses of the effect of dabigatran.

\section{Heptest Coagulation Assay}

Recently, the effect of dabigatran was investigated for the Heptest-STAT coagulation assay demonstrating a prolongation over a concentration range from approximately 50 to 500 $\mathrm{ng} / \mathrm{mL}$ of the anticoagulant. ${ }^{18}$ The assay is less sensitive at concentrations of dabigatran below 100 or $300 \mathrm{ng} / \mathrm{mL}$ and can be used if overdosing of dabigatran is suspected. These limitations in this assay are less pronounced for oral direct factor Xa inhibitors, which more specifically prolong this assay (see later).

\section{Prothrombinase-Induced Clotting Time}

The prothrombinase-induced clotting time (PiCT) assay uses prothrombinase to modify factor $\mathrm{V}$ and consequently factor $\mathrm{X}$ and prothrombin to activated clotting factors. Therefore, PiCT is sensitive to unfractionated heparins, low-molecularweight heparins, danaparoid, fondaparinux, hirudins, direct thrombin, and direct factor Xa inhibitors. For specific indirect and direct factor Xa inhibitors, the test has to be performed as a two-step method (reagents from Pentapharm, Basle, Switzerland) (normal mean value $29.9 \pm 4.6$ seconds $^{19}$ ). For other coagulation inhibitors, preincubation of plasma with prothrombinase is not required. ${ }^{20}$

\section{Prothrombin Time and Dilute Prothrombin Time Assays}

PT assays can be performed with many thromboplastin reagents. It has been demonstrated in several studies that every thromboplastin possesses an individual sensitivity toward dabigatran. A standardization of the reagents in relation to dabigatran has not been investigated. Importantly, the international normalized ratio (INR) cannot be used to standardize the influence of dabigatran on the PT as it was done for patients on treatment with coumarins. ${ }^{21,22}$ This is due to the fact that standardization of INR requires plasma samples of patients treated with coumarin, which is depletion of the vitamin K-dependent clotting factors. In contrast, plasma of patients treated with dabigatran contains normal concentration of these clotting factors rendering a calculation of the INR as inappropriate.

\section{Activated Partial Thromboplastin Time}

As for PT reagents, aPTT can be performed by using several commercially available activators for the assay. Transformation of the clotting times to ratios may be helpful to compare the effects of dabigatran, but relationships between concentrations and ratios still vary between reagents. Further standardization activities have not been published for aPTT reagents. All aPTT reagents are insensitive to low concentrations of dabigatran. High concentration of dabigatran in plasma prolong coagulation times of aPTT, but the coefficient of variation remains above $50 \%$ even for plasma samples spiked with dabigatran and analyzed within 1 day (intraday variation). ${ }^{23-26}$ For clinical use, large prolongations of aPTT indicate high concentrations of dabigatran in plasma. However, the variation of the results is too wide to sensitively detect overdosing of dabigatran. This underpins the limited value of aPTT for the determination of dabigatran in plasma samples of patients treated with this anticoagulant.

\section{Chromogenic Assays for Oral Direct Factor Xa Inhibitors}

Several chromogenic substrates have been proven to specifically determine the inhibition of rivaroxaban, apixaban, edoxaban, and other oral direct factor Xa inhibitors in human plasma samples. The dependence of each chromogenic method on the coagulation platform used with regard to the measured concentration of apixaban was described above. These findings should be applicable to other oral factor Xa inhibitors as well. The chromogenic substrates differ in their affinity, association, and dissociation constants toward factor Xa. Consequently, the kinetics of interaction are also influenced differently by the factor Xa inhibitors. This was investigated in detail for rivaroxaban using five chromogenic substrates. ${ }^{27}$ Human plasma samples were spiked with various concentrations of rivaroxaban and incubated with the chromogenic substrate, and the release of para-nitroaniline by the residual activity of factor Xa was determined. The optical density (OD at $405 \mathrm{~nm}$ ) was plotted versus the concentration of rivaroxaban in the sample. The results demonstrated significant differences between the OD and concentration curves of the chromogenic substrates. By statistical methods taking these differences into account, overlapping OD versus concentration curves for rivaroxaban with the various chromogenic substrates were obtained. ${ }^{27}$ By this procedure, the variability between assay results was dramatically reduced. The application of this method to clinical samples remains to be validated. These data demonstrate that differences between results of chromogenic substrate assays can be eliminated by mathematical approaches. Careful adaptation of chromogenic methods to hemostasis instrument platforms may reduce this variability further.

\section{Clotting Assays for Measurement of Oral Direct Factor Xa Inhibitors}

\section{Heptest Coagulation Assay}

Heptest STAT is a coagulation assay that is activated by a reagent containing calcium, phospholipids, and factor $\mathrm{V}$ (HemaXa Ltd, Tioga, TX). The previously available Heptest coagulation assay sensitively determines the activities of unfractionated heparins, ${ }^{28,29}$ low-molecular-weight heparins, $^{30}$ fondaparinux, ${ }^{31}$ and heparinoids. ${ }^{32}$ Heptest was used in phase $\mathrm{I}^{33}$ phase II, or phase III clinical trials to determine the pharmacodynamics effects of rivaroxaban ${ }^{34}$ 
and apixaban. ${ }^{35}$ The utility of Heptest STAT under real-life conditions has so far only been examined in patients treated with low-molecular-weight heparins. ${ }^{36}$ A recent investigation demonstrated that Heptest STAT determined the concentrations of the NOACs rivaroxaban, apixaban, and dabigatran as accurately as specific chromogenic assays. ${ }^{18}$

\section{Prothrombinase-Induced Clotting Time}

The PiCT assay is sensitive toward rivaroxaban and other direct factor Xa inhibitors if plasma samples are preincubated with prothrombinase. ${ }^{37}$ Newer publications on the influence of NOAC on PiCT are not available.

\section{Prothrombin Time and Dilute Prothrombin Time}

PT is prolonged dose dependently by all thromboplastin reagents. The sensitivity of the thromboplastin reagents varies substantially for the different direct factor Xa inhibitors. In addition, the factor Xa inhibitors differ substantially in their inhibitory potency as determined by PT with rivaroxaban being the most potent and apixaban the least potent agent in this assay. ${ }^{38,39}$ The results from clinical trials vary substantially. A dilution of the thromboplastin reagent did not improve the sensitivity of the reagent toward the factor Xa inhibitors. From a clinical point of view, the variability of the response of thromboplastin reagents is too high to accurately identify low or high concentrations of rivaroxaban. ${ }^{40}$

\section{Determination of NOAC from Plasma Samples Anticoagulated with EDTA}

Sodium citrate is routinely added to blood samples to prevent blood coagulation in vitro for coagulation assays, and ethylene diamine tetraacetic acid (EDTA) is added for determination of hematology and some clinical chemistry parameters. In contrast to plasma obtained with citrate, EDTA plasma specimens cannot be accurately tested by clotting-based assays due to the irreversible chelation of ionic calcium. While clotting assays require ionized calcium for the enzymatic reaction, this may not be the case for the interaction of factor Xa and a chromogenic substrate. ${ }^{39}$ Recent results demonstrated that rivaroxaban determinations in plasma samples anticoagulated with sodium citrate or EDTA and spiked with serial dilutions of rivaroxaban were not statistically significantly different for two chromogenic assays. For patients on treatment with rivaroxaban, the original values for mean concentrations and variances of rivaroxaban were also not different in samples anticoagulated by either sodium citrate or EDTA during sampling. ${ }^{41}$ Additional data need to be generated with other direct factor Xa inhibitors and by increasing the number of patients on treatment. However, this approach is promising and of clinical relevance, if only EDTA, but no citrate plasma, samples are available from patients.

\section{Point-of-Care Tests Using Plasma Samples}

To determine the presence of NOAC in blood samples in emergency situations, POC tests from blood may offer advantages over conventional clotting assays such as PT or aPTT. It has to be considered that results of a POC test are influenced by the sensitivity of the reagents toward the NOAC and by the POC platform. This has been described in detail earlier for apixaban and a chromogenic test. At present, one publication is available with results for dabigatran and rivaroxaban determined on two POC devices. ${ }^{42}$ The results show a high variability of the results with both anticoagulants after adopting an aPTT reagent for measuring dabigatran and two PT thromboplastin reagents for rivaroxaban. The authors concluded that both methods may be useful for detecting an overdose of dabigatran and rivaroxaban in acute clinical conditions. $^{42}$ Additional publications are currently not available.

\section{Determination of NOAC from Serum Samples}

Unlike plasma samples, serum samples are taken from all patients in acute and nonacute clinical situations. Blood for the determination of coagulation parameters needs to be collected separately into plastic or siliconized glass tubes containing sodium citrate to inhibit mechanical coagulation activation in vitro. Potential pitfalls of blood drawing for coagulation parameters are incomplete filling of the tube resulting in wrong ratios of anticoagulant to blood leading to incorrect coagulation results and the activation of blood clotting during and after blood sampling due to incorrect handling. In addition, correct handling also includes centrifugation at given temperatures, analysis within a given time frame, and specific procedures for freezing and thawing of samples. ${ }^{43}$ Published data indicate that rivaroxaban and apixaban can be accurately determined from serum samples of patients on treatment and that values correlate well with those determined from plasma samples. ${ }^{44}$ This would seem advantageous given the wider availability of serum samples compared with plasma samples, and the easier handling/ preanalytical stability of serum samples.

In contrast, however, other data indicate that dabigatran cannot be accurately determined from serum samples. The variability of the lower values of dabigatran in serum compared with plasma was twice as high using chromogenic assays. An explanation for this finding includes a consumption of dabigatran during thrombin generation of blood samples to obtain serum. The determination from serum still requires improvement before a general applicability in patients may be achieved. ${ }^{13}$

\section{Measurement of NOAC from Urine Samples}

As stated earlier, the elimination of NOAC variably decreases with decreasing renal function. Therefore, patients on treatment with NOAC and with impaired renal function may be exposed to an increased risk of bleeding due to drug accumulation. ${ }^{2}$ For example, the mean clearance of total dabigatran decreased from $62.9 \mathrm{~mL} / \mathrm{min}$ in the healthy control group to $10.3 \mathrm{~mL} / \mathrm{min}$ in subjects with severe renal impairment. The mean elimination half-life of dabigatran increased from 13.8 hours in healthy subjects to $16.6,18.7$, and 27.5 hours in subjects with mild, moderate, and severe renal impairment, respectively (-Table 1). 
Table 1 Ratios of $C_{\max }$ and AUC ( $\mathrm{ng} \times \mathrm{h} / \mathrm{mL}$ ) for samples from patients with renal impairment and those from healthy subjects following a single oral administration of dabigatran ${ }^{45}$

\begin{tabular}{|l|l|l|}
\hline Parameter & Renal impairment & Ratio $(90 \% \mathrm{Cl})$ \\
\hline $\mathrm{C}_{\max }(\mathrm{ng} / \mathrm{mL})$ & Mild & $1.11(0.61-2.03)$ \\
\cline { 2 - 3 } & Moderate & $1.70(0.93-3.10)$ \\
\cline { 2 - 3 } & Severe & $2.12(1.25-3.59)$ \\
\hline \multirow{2}{*}{ AUC $(\mathrm{ng} \times \mathrm{h} / \mathrm{mL})$} & Mild & $1.50(0.78-2.90)$ \\
\cline { 2 - 3 } & Moderate & $3.15(1.63-6.08)$ \\
\cline { 2 - 3 } & Severe & $6.31(3.54-11.25)$ \\
\hline
\end{tabular}

Abbreviations: AUC, area under the concentration time curve; $\mathrm{Cl}$, confidence interval; $C_{\max }$, maximal concentration.

The determination of dabigatran in urine showed high concentrations in the range of 2 to $20 \mu \mathrm{g} / \mathrm{mL} .^{13}$ The value of measuring dabigatran in urine is that this method is not invasive, may be repeated easily, and detect patients with a lack of compliance even if considered as a semiquantitative assessment. The measurement of dabigatran in urine is feasible and may be used to check compliance as has been already demonstrated with a qualitative test. ${ }^{9}$ The validity of the assay results remains to be determined in specific clinical settings under real-life conditions of therapy. Similar reports are not yet available for rivaroxaban and apixaban.

\section{Point-of-Care Test for NOAC from Urine Samples}

Development of POC methods for DOACs in urine is based on their immediate glomerular filtration and excretion into the urine if present in blood. The test principle is based on the development of different colors in the presence and absence of oral direct factor Xa or thrombin inhibitors, and which can be identified by the naked eye. Two colors were chosen for each class of inhibitors: for factor Xa (absence: yellow color; presence: no color; -Fig. 1) and for thrombin inhibitors (absence: green color; presence: blue color; - Fig. 1). ${ }^{46}$

The ability of patients to correctly identify the colors with the naked eye as being positive and negative for dabigatran and rivaroxaban was analyzed by a set of prepared samples obtained from patients under therapy. The results of the sensitivity, specificity, accuracy, and positive and negative predictive indices are given in - Table $2 .{ }^{46}$

In specific clinical situations, the rapid determination of NOAC may be required for clinical decision making. Typical examples are where it is necessary to know if the drug is on board or not, or there are indications for fibrinolytic therapy in cerebral embolism, for placement of a lumbar catheter, and/or severe bleeding events. Renal function reduces with increasing age. Advantages of using urine as test sample include its noninvasive availability and the possibility to develop POC test systems to be performed by trained personal or by the patients themselves. These tests can be repeated easily. In contrast, POC tests from blood require blood sampling from the fingertip and accurate administration to the test strip of the coagulation device. In addition, standardization of the POC test is not required for urine samples, as sensitivity and specificity of the NOAC tests in urine have homogeneously been found to be above $95 \% .{ }^{46}$ A scheme for the clinical disease management of specific patient groups is given in - Table 3 in relation to the results of the qualitative POC test for NOAC.

\section{Discussion}

Several chromogenic assays and clotting tests were evaluated in the last couple of years to measure the anticoagulant effects of NOAC in specific patient populations. ${ }^{47}$ Specific problems were identified in these investigations. Standardization of assays for each platform is required to improve comparability of the measurements and related publications. It has still to be stressed that INR values as being specifically designed and standardized with plasma samples of patients treated with vitamin $\mathrm{K}$ antagonist are unsuitable for analyzing the effects of NOAC. Specific liquid chromatography methods are not suitable for routine monitoring due to the sophisticated equipment required and the specific knowledge of specialists to run these assays. Of note, comparisons between these test systems remain to be performed. On the other hand, high correlations of the LC-MS/MS methods with chromogenic assays and Hemoclot limit the necessity to perform these tests for experiments in the future.

Table 2 Sensitivity, specificity, NPV, and PPV of the POC method using urine samples of patients on treatment as determined by color reading by patients 46

\begin{tabular}{|l|l|l|l|l|}
\hline \multirow{2}{*}{} & \multicolumn{3}{|l|}{ Dabigatran $(\boldsymbol{n}=\mathbf{4 8 4})$} & \multicolumn{2}{l|}{ Rivaroxaban $(\boldsymbol{n}=\mathbf{4 5 7})$} \\
\cline { 2 - 5 } & Mean & $95 \% \mathrm{Cl}$ & Mean & $95 \% \mathrm{Cl}$ \\
\hline Sensitivity & 100 & $99-100$ & 96 & $94-98$ \\
\hline Specificity & 99 & $98-100$ & 98 & $96-99$ \\
\hline Accuracy & 99 & $99-100$ & 97 & $96-98$ \\
\hline PPV & 99 & $98-100$ & 98 & $97-99$ \\
\hline NPV & 100 & $99-100$ & 96 & $94-98$ \\
\hline
\end{tabular}

Abbreviations: $\mathrm{Cl}$, confidence interval; NPV, negative predictive value; POC, point of care; PPV, positive predictive value. 
Table 3 Position of the urinary POC test results in the context of clinical disease management

\begin{tabular}{|c|}
\hline Bleeding complication \\
\hline Test is negative: \\
\hline • Patient did not take medication within the past 1 -24 $\mathrm{h}$ \\
\hline \\
• Stop bleeding, re-check POC after $1 \mathrm{~h}$, determine \\
chromogenic test
\end{tabular}

Abbreviations: $\mathrm{ECT}$, ecarin clotting time; NOAC, non-vitamin $\mathrm{K}$ antagonist oral anticoagulant; POC, point of care.

Several recommendations or guidelines have been published on the use of coagulation assays to monitor NOAC. ${ }^{10,16,40,48}$ However, careful reading of the recommendations may also convey the impression that a consensus on this topic is not yet obtained. ${ }^{38}$

A major limitation of all available methods is the fact that a wide range of values is obtained from patients under treatment and consequently essentially overlap in values exists between controls and treated patients. Clinical decision making may thus be difficult in relation to the obtained coagulation values. Therefore, efforts are undertaken to tackle this unmet medical need of accurately and feasibly measuring the anticoagulant effects of NOAC. So far, only the determination of NOAC in urine samples offers a possibility to partly address this problem, which is related to the high rate of renal excretion of these anticoagulants reaching urinary concentrations of above $100 \mathrm{ng} / \mathrm{mL}$ as soon as a NOAC is present in blood. Thus, an overlap with controls was not observed with such testing. ${ }^{13}$ These assays were also developed as qualitative POC methods using urine samples, which can be read by the naked eye within a couple of minutes by different colors for a negative and positive result (absence and presence of the NOAC). ${ }^{46}$ One limitation of these methods is the time difference of approximately 1 to 2 hours between actual concentration of NOAC in blood and appearance in urine. Strengths of the methods are that they are not invasive, can be repeated easily, they are highly sensitive and specific, and that the POC test can be performed by the patients themselves. Further development of the test system is currently on the way at specialized centers.

In conclusion, current methods to measure NOAC in several matrices are chromogenic substrate assays and some clotting methods. POC methods are developed to speed up the decision making in critical care medicine. There is a high possibility of solving the current methodological problems and limitations of the assays due to an intense collaboration of producers of the drugs, of the assay reagents and coagulation platforms, laboratory and clinical scientists, and physicians.

\section{Conflict of Interest}

J.H.: Lecturing and consulting fees from Novartis, Bayer HealthCare, Boehringer Ingelheim, Roche Diagnostics, Pfizer, Bristol-Myers Squibb, Daichii-Sankyo, LEO Pharma; support of research from Novartis, Bayer HealthCare, Boehringer Ingelheim, Roche Diagnostics, Pfizer, BristolMyers Squibb, HepLabs.

M.W.: Employed by AstraZeneca R\&D, Mölndal, as director of discovery medicine (= translational medicine) from 2004 to 2006, while on sabbatical leave from his professorship at the University of Heidelberg. After return to this position in January 2007, he received lecturing and consulting fees from Novartis, Pro Bono Bio, Bayer HealthCare, Boehringer Ingelheim, Roche, Pfizer, Bristol-Myers Squibb, Daichii-Sankyo, LEO, Lilly, Shire, Mundipharm, and NovoNordisk.

R.K.: Support of research from Medinvent, Siemens Healthcare. The other authors do not have to declare a conflict of interest.

\section{Acknowledgments}

The authors thank the technical assistance of Mrs. Christina Giese, Antje Hagedorn, and Inge Träger. The research was supported by a grant from the Dietmar-Hopp Foundation to J.H. 


\section{References}

1 Hylek EM, Ko D, Cove CL. Gaps in translation from trials to practice: non-vitamin K antagonist oral anticoagulants (NOACs) for stroke prevention in atrial fibrillation. Thromb Haemost 2014;111(5): 783-788

2 Harenberg J, Wehling M. Future of anticoagulant therapy. Cardiovasc Ther 2011;29(5):291-300

3 Antovic JP, Skeppholm M, Eintrei J, et al. Evaluation of coagulation assays versus LC-MS/MS for determinations of dabigatran concentrations in plasma. Eur J Clin Pharmacol 2013;69(11):1875-1881

4 Douxfils J, Dogné JM, Mullier F, et al. Comparison of calibrated dilute thrombin time and aPTT tests with LC-MS/MS for the therapeutic monitoring of patients treated with dabigatran etexilate. Thromb Haemost 2013;110(3):543-549

5 Harenberg J, Kraemer S, Du S, et al. Determination of direct oral anticoagulants from human serum samples. Semin Thromb Hemost 2014;40(1):129-134

6 Gous T, Couchman L, Patel JP, Paradzai C, Arya R, Flanagan RJ. Measurement of the direct oral anticoagulants apixaban, dabigatran, edoxaban, and rivaroxaban in human plasma using turbulent flow liquid chromatography with high-resolution mass spectrometry. Ther Drug Monit 2014;36(5):597-605

7 Schmitz EM, Boonen K, van den Heuvel DJ, et al. Determination of dabigatran, rivaroxaban and apixaban by ultra-performance liquid chromatography - tandem mass spectrometry (UPLC-MS/MS) and coagulation assays for therapy monitoring of novel direct oral anticoagulants. J Thromb Haemost 2014;12(10):1636-1646

8 Delavenne X, Moracchini J, Laporte S, Mismetti P, Basset T. UPLC MS/MS assay for routine quantification of dabigatran - a direct thrombin inhibitor - in human plasma. J Pharm Biomed Anal 2012; 58:152-156

9 Harenberg J, Du S, Krämer S, et al. Novel methods for assessing oral direct factor Xa and thrombin inhibitors: use of point-of-care testing and urine samples. Semin Thromb Hemost 2013;39(1):66-71

10 Harenberg J, Du S, Weiss C, Krämer R, Hoppensteadt D, Walenga J; working party: methods to determine apixaban of the Subcommittee on Control of Anticoagulation of the International Society of Thrombosis and Haemostasis. Report of the Subcommittee on Control of Anticoagulation on the determination of the anticoagulant effects of apixaban: communication from the SSC of the ISTH. J Thromb Haemost 2014;12(5):801-804

11 Hauel NH, Nar H, Priepke H, Ries U, Stassen JM, Wienen W. Structure-based design of novel potent nonpeptide thrombin inhibitors. J Med Chem 2002;45(9):1757-1766

12 Stangier J, Stähle H, Rathgen K, Roth W, Reseski K, Körnicke T. Pharmacokinetics and pharmacodynamics of dabigatran etexilate, an oral direct thrombin inhibitor, with coadministration of digoxin. J Clin Pharmacol 2012;52(2):243-250

13 Du S, Weiss C, Giese C, et al. Determination of dabigatran in plasma, serum and urine samples: comparison of six methods. Clin Chem Lab Med. In press

14 Harenberg J, Giese C, Marx S, Krämer R. Determination of dabigatran in human plasma samples. Semin Thromb Hemost 2012; 38(1):16-22

15 Nowak G. The ecarin clotting time, a universal method to quantify direct thrombin inhibitors. Pathophysiol Haemost Thromb 2003; 33(4):173-183

16 Fenyvesi T, Jörg I, Weiss C, Harenberg J. Effects of lepirudin, argatroban and melagatran and additional influence of phenprocoumon on ecarin clotting time. Thromb Res 2003;111(1-2):89-94

17 Stangier J, Feuring M. Using the HEMOCLOT direct thrombin inhibitor assay to determine plasma concentrations of dabigatran. Blood Coagul Fibrinolysis 2012;23(2):138-143

18 Harenberg J, Du S, Krämer S, Krämer R, Wehling M, Weiss C. Measurement of non-vitamin $\mathrm{K}$ antagonist oral anticoagulants in patient plasma using Heptest-STAT coagulation method (epub ahead of print). Ther Drug Monit 2014
19 Harenberg J, Giese C, Hagedorn A, Traeger I, Fenyvesi T. Determination of antithrombin-dependent factor Xa inhibitors by prothrombin-induced clotting time. Semin Thromb Hemost 2007; 33(5):503-507

20 Calatzis A, Peetz D, Haas S, Spannagl M, Rudin K, Wilmer M. Prothrombinase-induced clotting time assay for determination of the anticoagulant effects of unfractionated and low-molecularweight heparins, fondaparinux, and thrombin inhibitors. Am J Clin Pathol 2008;130(3):446-454

21 Samama MM, Martinoli JL, LeFlem L, et al. Assessment of laboratory assays to measure rivaroxaban-an oral, direct factor Xa inhibitor. Thromb Haemost 2010;103(4):815-825

22 Tripodi A, Chantarangkul V, Guinet C, Samama MM. The International Normalized Ratio calibrated for rivaroxaban has the potential to normalize prothrombin time results for rivaroxaban-treated patients: results of an in vitro study. J Thromb Haemost 2011;9(1): 226-228

23 Van Blerk M, Bailleul E, Chatelain B, et al. Influence of dabigatran and rivaroxaban on routine coagulation assays. A nationwide Belgian survey. Thromb Haemost 2014;112(6):

24 Hapgood G, Butler J, Malan E, Chunilal S, Tran H. The effect of dabigatran on the activated partial thromboplastin time and thrombin time as determined by the Hemoclot thrombin inhibitor assay in patient plasma samples. Thromb Haemost 2013;110(2): 308-315

25 Samama MM, Contant G, Spiro TE, et al. Laboratory assessment of rivaroxaban: a review. Thromb J 2013;11(1):11

26 Suzuki S, Otsuka T, Sagara K, et al. Dabigatran in clinical practice for atrial fibrillation with special reference to activated partial thromboplastin time. Circ J 2012;76(3):755-757

27 Harenberg J, Krämer R, Giese C, Marx S, Weiss C, Wehling M. Determination of rivaroxaban by different factor Xa specific chromogenic substrate assays: reduction of interassay variability. J Thromb Thrombolysis 2011;32(3):267-271

28 Emanuele RM, Racanelli A, Fareed J. Pharmacokinetics of heparins differing in mean molecular weight using a Xa amidolytic and Heptest clotting method. Ther Drug Monit 1988;10(2):153-159

29 Yin ET, Wessler S, Butler JV. Plasma heparin: a unique, practical, submicrogram-sensitive assay. J Lab Clin Med 1973;81(2): 298-310

30 Hoffmann U, Harenberg J, Bauer $\mathrm{K}$, et al. Bioequivalence of subcutaneous and intravenous body-weight-independent highdose low-molecular-weight heparin Certoparin on anti-Xa, Heptest, and tissue factor pathway inhibitor activity in volunteers. Blood Coagul Fibrinolysis 2002;13(4):289-296

31 Jeske WP, Walenga JM, Samama MM, Hoppensteadt D, Mayuga M, Fareed J. Functionality of fondaparinux (pentasaccharide) depends on clinical antithrombin levels. Blood Coagul Fibrinolysis 2011; 22(3):206-210

32 Harenberg J, Jeschek M, Acker M, Malsch R, Huhle G, Heene DL. Effects of low-molecular-weight dermatan sulfate on coagulation, fibrinolysis and tissue factor pathway inhibitor in healthy volunteers. Blood Coagul Fibrinolysis 1996;7(1):49-56

33 Kubitza D, Becka M, Mück W, Krätzschmar J. Pharmacodynamics and pharmacokinetics during the transition from warfarin to rivaroxaban: a randomized study in healthy subjects. Br J Clin Pharmacol 2014;78(2):353-363

34 Mueck W, Lensing AW, Agnelli G, Decousus H, Prandoni P, Misselwitz F. Rivaroxaban: population pharmacokinetic analyses in patients treated for acute deep-vein thrombosis and exposure simulations in patients with atrial fibrillation treated for stroke prevention. Clin Pharmacokinet 2011;50(10):675-686

35 Miyares MA, Davis K. Newer oral anticoagulants: a review of laboratory monitoring options and reversal agents in the hemorrhagic patient. Am J Health Syst Pharm 2012;69(17):1473-1484

36 Dempfle CE, Zharkowa U, Elmas E, Ahmad-Nejad P, Neumaier M, Borggrefe M. Heptest-STAT, a new assay for monitoring of low- 
molecular-weight heparins, is not influenced by pregnancy-related changes of blood plasma. Thromb Haemost 2009;102(5):1001-1006

37 Kluft C, Meijer P, Kret R, Burggraaf J. Preincubation in the Prothrombinase-induced Clotting Time test ( $\mathrm{PiCT}$ ) is necessary for in vitro evaluation of fondaparinux and to be avoided for the reversible, direct factor Xa inhibitor, rivaroxaban. Int J Lab Hematol 2013;35(4):379-384

38 Lippi G, Favaloro EJ. Recent guidelines and recommendations for laboratory assessment of the direct oral anticoagulants (DOACs): is there consensus? Clin Chem Lab Med 2014

39 Rosén S. Chromogenic methods in coagulation diagnostics. Hamostaseologie 2005;25(3):259-266

40 Douxfils J, Mullier F, Loosen C, Chatelain C, Chatelain B, Dogné JM. Assessment of the impact of rivaroxaban on coagulation assays: laboratory recommendations for the monitoring of rivaroxaban and review of the literature. Thromb Res 2012;130(6):956-966

41 Du S, Krämer S, Giese C, Weiss C, Wehling M, Krämer R, Harenberg $\mathrm{J}$. Chromogenic assays for measurement of rivaroxaban from EDTA anticoagulated plasma samples (epub ahead of print) Thromb Haemost 2015;113(5)

42 Mani V, Wang S, Inci F, De Libero G, Singhal A, Demirci U. Emerging technologies for monitoring drug-resistant tuberculosis at the point-of-care. Adv Drug Deliv Rev 2014;78C:105-117
43 Lippi G, Salvagno GL, Montagnana M, Lima-Oliveira G, Guidi GC, Favaloro EJ. Quality standards for sample collection in coagulation testing. Semin Thromb Hemost 2012;38(6):565-575

44 Harenberg J, Krämer S, Du S, et al. Measurement of rivaroxaban and apixaban in serum samples of patients. Eur J Clin Invest 2014; 44(8):743-752

45 Stangier J, Rathgen K, Stähle H, Mazur D. Influence of renal impairment on the pharmacokinetics and pharmacodynamics of oral dabigatran etexilate: an open-label, parallel-group, single-centre study. Clin Pharmacokinet 2010;49(4):259-268

46 Harenberg J, Krämer S, Du S, Weiss C, Krämer R. Concept of a point of care test to detect new oral anticoagulants in urine samples. Thromb J 2013;11(1):15

47 Cuker A, Siegal DM, Crowther MA, Garcia DA. Laboratory measurement of the anticoagulant activity of the non-vitamin $\mathrm{K}$ oral anticoagulants. J Am Coll Cardiol 2014;64(11): 1128-1139

48 Baglin T, Hillarp A, Tripodi A, Elalamy I, Buller H, Ageno W. Measuring Oral Direct Inhibitors (ODIs) of thrombin and factor Xa: A recommendation from the Subcommittee on Control of Anticoagulation of the Scientific and Standardisation Committee of the International Society on Thrombosis and Haemostasis. J Thromb Haemost 2013;11(4):756-760 